\title{
A Comparison of the Impact of Avoidance Cues in Hazard Avoidance During Evaluation of Text Entry
}

\author{
Joanna Lumsden and Patrick Drost \\ National Research Council of Canada \\ 46 Dineen Drive, Fredericton, N.B., \\ Canada E3B 9W4 \\ 1.506.444.0382 \\ jo.lumsden@nrc-cnrc.gc.ca
}

\begin{abstract}
The research presented in this paper is part of an ongoing investigation into how best to support meaningful lab-based evaluations of mobile technologies. In our previous work, we developed a hazard avoidance system for use during lab evaluations [1]; in the work reported here, we further assess the impact of this system, specifically in terms of the effect of avoidance cue type on speech-based text entry tasks.
\end{abstract}

\section{Categories and Subject Descriptors}

H.5.2 [Information Interfaces and Presentation (e.g., HCI)]: User Interfaces - Evaluation/methodology; Voice I/O.

\section{General Terms}

Performance, Experimentation, Human Factors.

\section{Keywords}

Mobile technology, mobile lab-based evaluation technique.

\section{INTRODUCTION}

For mobile applications, the benefits of lab evaluations over field evaluations are subject to much debate [e.g., 3, 7]. Lab evaluations facilitate easier data collection and increased environmental control; field evaluations increase the realism of the evaluation context (although the mechanics of conducting an 'in the wild' evaluation can ultimately impede the realism [4]). Although we feel there is a place for both types of evaluation during the design of a mobile application, our focus in this paper is on improving lab-based studies such that they are better equipped to mimic the real world contexts in which mobile technologies may be used, and thereby better positioned to return meaningful evaluation results.

In the real world, users of mobile devices are typically required to simultaneously monitor and navigate through their physical environment while avoiding potential hazards. Increasingly, lab evaluations of mobile applications are incorporating mobility [e.g., 4, 9], but they typically fail to require that participants monitor their environment or to navigate a truly dynamic path. This reduces the effect of the mobility to the impact of the motion, and ignores other factors such as the

(C) The Author 2008 .

Published by the British Computer Society cognitive and visual demands typically placed on users in the real world.

We have developed a novel 'hazard avoidance' system for use in lab-based evaluations of mobile applications [1]: the technique requires that participants monitor their environment and change the route they are walking to avoid dynamically changing hazards, much as real world users would be required to do. The research discussed in this short paper represents a combination and extension of work we presented at HCI'2007 $[1,5]$ : we previously introduced the technique and discussed a series of experiments in which it was used, demonstrating that our technique is capable of being used to create an appropriate context in which to evaluate mobile systems in a lab. Space does not permit us to elaborate here on the background to our work or to discuss the evolution of our technique; we would, instead, refer interested readers to $[1,5]$ for such information.

In this paper, we report on an empirical study we conducted to compare the use of a textual avoidance cue to a symbolic avoidance cue within our hazard avoidance system, in order to determine the impact of each on participants' capacity to correctly enter textual data (using speech) into a mobile application. In Section 2, we briefly describe our hazard avoidance technique (more detail is available in [1]). In Section 3, we describe, in detail, our study design before presenting and discussing our results in Section 4. This paper closes with conclusions and a discussion of further work.

\section{HAZARD AVOIDANCE TECHNIQUE}

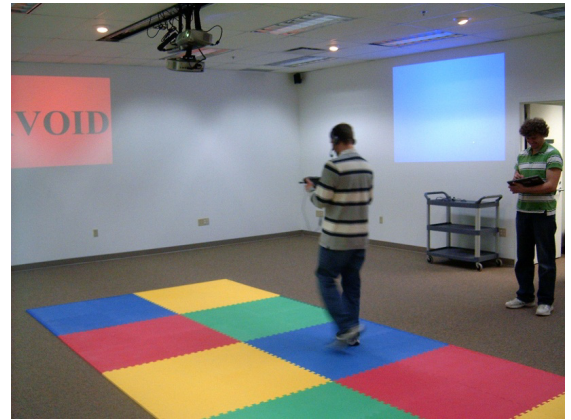

Figure 1: Hazard avoidance system in use.

Figure 1 shows the hazard avoidance system in use. The system consists of a grid of colored mats (red, green, yellow, and blue), arranged in 4' x 4' colored squares, and organized in such a way that no two squares of the same color are adjacent. The squares are arranged on the floor of the lab in a grid that is 2 squares ( $\left.8^{\prime}\right)$ wide and 10 squares (40') long. The system also comprises a series of 6 ceiling mounted projectors, with 3 directed to the left, right, and centre walls at each end of the lab space. These projectors display a changing sequence of squares 
of colors corresponding to the mat colors; at any point in time, at most one of the projected squares (on the three walls facing the participant) will contain the word "Avoid". Whilst walking up and down the grid of mats, participants are required to monitor the projections to identify which (if any) color they are to avoid, and then avoid stepping on that color as they walk. This creates a dynamically changing environment and generates the need for participants to walk a dynamically changing path; it prevents participants from simply memorizing the path they are to walk and forces them to remain actively cognizant of their physical environment.

\section{EVALUATION DESIGN \& PROCESS}

As previously mentioned, the goal of our study was to investigate the extent (if any) to which the use of a textual avoidance cue ("Avoid"), as compared to a symbolic avoidance cue (" $\square$ "), within the hazard avoidance system impacts on participants' ability to correctly and efficiently enter textual data, using speech, into a mobile device while mobile. On the basis that we felt the textual avoidance cue would place greater cognitive demands on the participants than the symbolic cue due to potential for interference between the cue itself and data items, we hypothesized that participants' text entry performance would be better when exposed to the latter than the former.

As the basis for our study, we used the same simple data input application as we used in our previous study [5], adjusting the grammar to meet our needs (see later). The application was designed to run on a tablet PC running Windows XP and used the IBM ViaVoice speech recognition engine. We selected to use the Plantronics DSP-500 microphone on the basis that it was proven most effective in our previous study.

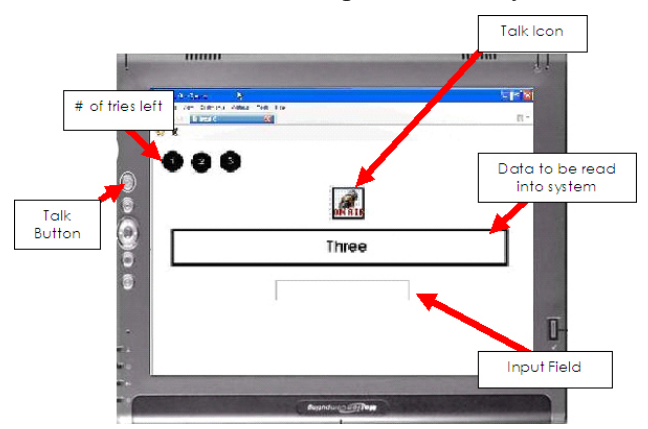

Figure 2: A screen dump of the evaluation application.

Figure 2 shows an annotated screen dump of the evaluation application. When participants pushed the 'talk button' an "on air" 'talk icon' was displayed to reinforce the fact that the system was ready to receive input. Participants were shown the 'data to be read into the system' and were required to input that item using speech. The results of their speech input were reflected in the 'input field'. Participants were given up to three attempts to achieve an accurate entry, the number of 'tries left' being shown at all times; upon an accurate entry and/or after participants had exhausted all tries for a given item, the system automatically moved them on to the next data input item. Participants were given training on how to use speech to enter data prior to commencing the study tasks; they were trained in conditions commensurate with those used in the study sessions themselves.

The grammar (word list) we chose for our study was drawn from the phrase sets developed by MacKenzie and Soukeroff [6]. In essence, we selected 40 words from their phrase sets such that $50 \%$ of words were similar in length, syllables, rhyme, and/or letters to the textual cue "Avoid", and 50\% were dissimilar.

We adopted a between-groups design, assigning participants to one of the two versions of the hazard avoidance system: Group 1 was exposed to the textual avoidance cue; Group 2 was exposed to the symbolic avoidance cue. During the experiment, a range of measures was taken to assess the impact of the avoidance cue on the efficiency and accuracy with which participants could enter the 40 data items using speech. We recorded the length of time participants took to complete their data entry tasks and the details of the data they entered. We also recorded their walking speed - normal (when not avoiding hazards or completing data entry tasks), when avoiding hazards but not completing data entry tasks, and when performing the study tasks while avoiding hazards - to allow us to determine the impact of use of the hazard avoidance system, and interaction with the technology, on walking speed. We recorded the number of hazardous steps made by participants that is, how often they stepped on a color actively cued as the hazard color - in order to determine the extent to which participants were environmentally aware as they performed their primary tasks. Additionally, we asked participants to indicate their subjective experience of workload (using the NASA TLX scales [2]).

Since studies have shown that speech recognition rates are typically much lower for accented speakers [8], we restricted our recruitment to participants who were native English speakers with a Canadian accent; we recruited equal numbers of male and female participants, and distributed both genders equally across our study groups. Due to the color-centric nature of our hazard avoidance system, we were not able to include color-blind participants. Sixteen people participated in our study, 8 per avoidance cue group.

\section{RESULTS \& DISCUSSION}

The following sections reflect on analysis of the measures we took to assess the impact of avoidance cue on participants' capacity to effectively and efficiently enter text using speech.

\subsection{Accuracy}

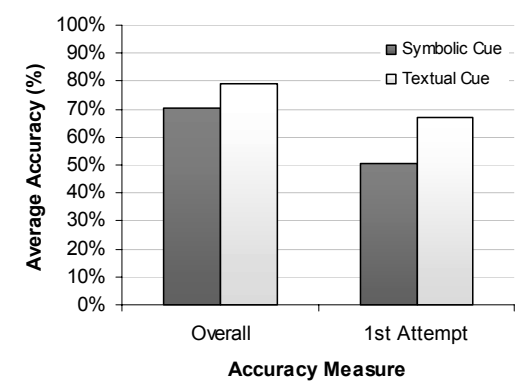

Figure 3: Average accuracy rates achieved according to measure and avoidance cue.

We adopted a simple measure of accuracy per data item: namely, ultimate success (a Boolean value of 1 for correct and 0 for incorrect after exhausting all available tries) divided by the number of tries used. A multiple factor ANOvA showed that avoidance cue had a significant effect $\left(\mathrm{F}_{1,636}=10.72, \mathrm{p}=0.001\right)$ on accuracy. As shown in Figure 3, participants exposed to the symbolic cue achieved an average overall accuracy rate of $70.3 \%$, compared to $79.4 \%$ achieved by participants exposed to the textual avoidance cue. This difference was not, however, at the expense of task completion time, there being no significant difference between the two groups in this respect. To observe 
the impact of avoidance cue on participants' initial attempt at each data entry, we adopted a second measure of accuracy based on the success of entry on participants' first attempt at each item. A multiple factor ANOVA again showed that avoidance cue had a significant effect $\left(\mathrm{F}_{1,636}=18.53, \mathrm{p}<0.001\right)$ on accuracy; participants exposed to the symbolic cue achieved a lower average first attempt accuracy rate (50.3\%) than participants exposed to the textual cue $(66.9 \%)$.

When considered in combination with the avoidance cue, the similarity of data items to the textual cue did not significantly impact accuracy rates. Finally, gender was not found to exert a significant influence on either measure of accuracy but, as illustrated in Figure 4, it is interesting to note that (albeit not statistically significant) the combination of gender and avoidance cue showed that males exposed to the symbolic cue performed worse (in terms of overall accuracy) than males exposed to the textual cue; the same degree of difference is not immediately apparent for females.

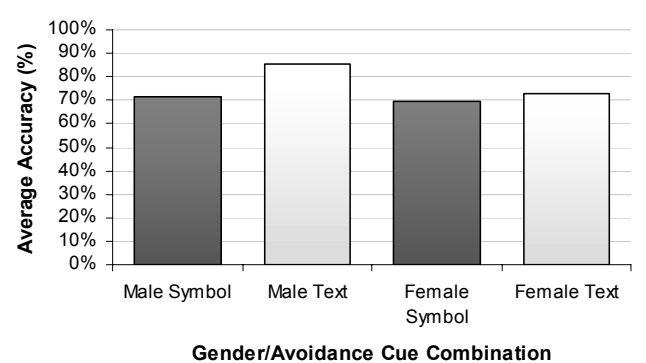

Figure 4: Average overall accuracy rates achieved according to gender and avoidance cue.

As previously noted, we had hypothesized that participants exposed to the symbolic avoidance cue would achieve higher accuracy rates than those exposed to the textual cue. On the basis of our accuracy results, however, our hypothesis would not appear to hold. According to both measures of accuracy, participants exposed to the textual avoidance cue actually performed significantly better; furthermore, participants using the textual cue ("Avoid") did not, contrary to our expectations, appear to be affected by the similarity of their data input items to the avoidance cue. It would seem, therefore, that the symbolic avoidance cue may actually be more cognitively demanding, perhaps as a result of the need to constantly switch from textual to image processing.

\subsection{Data Entry Error Types}

For each failed data entry attempt we analyzed and classified the type of error that led to the failure. We identified 5 classes of error: (a) clear - the participant's utterance was clear (to the human ear) but the speech recognition engine was unable to correctly interpret it; (b) distorted - the participant's speech was distorted in some way, making it hard for the speech recognition engine to disambiguate the signal; (c) error type 1 occurred when a participant simply said the wrong word (human error); (d) error type 2 - occurred when a participant said the correct word but pronounced it incorrectly (human error); and (e) error type 3 - occurred when a participant started speaking before fully engaging the microphone and/or disengaged the microphone before finishing speaking (essentially clipping the speech signal - again, human error). Avoidance cue had a significant impact on the clear errors made $\left(\mathrm{F}_{1,636}=8.92, \mathrm{p}=0.003\right)$; a greater percentage of these errors were attributable to participants exposed to the symbolic cue $(61.5 \%)$ compared to the textual cue $(38.5 \%)$. The combination of avoidance cue and gender had a significant impact on the number of type 3 errors $\left(\mathrm{F}_{1,636}=7.18, \mathrm{p}=0.008\right)$.
Tukey HSD tests revealed a significant difference $(\mathrm{p}=0.008)$ between males exposed to the textual avoidance cue $(12.9 \%$ of type 3 errors) compared to males exposed to the symbolic cue (30.9\% of type 3 errors); the same difference as not apparent for females. This suggests that males, in particular, appear to have experienced difficulty in terms of coordinating control of the microphone while simultaneously mobile, entering text, and interpreting symbolic cues (perhaps on account of the need to multitask and context switch from textual to graphical processing). Although females' incidence of type 3 errors was consistent across both avoidance cues, suggesting they remained unaffected by the need to context switch, they nevertheless made significantly more $(\mathrm{p}=0.039)$ type 3 errors when exposed to the textual avoidance cue $(29.9 \%)$ than males exposed to the same cue $(12.9 \%)$ This suggests that for females it was the use of the technology per se, as opposed to avoidance cue type, that affected their accuracy rates compared to males.

\subsection{Hazard Avoidance}

We used two measures to assess the extent to which participants successfully negotiated the avoidance of hazards as they completed their data entry tasks. The first measure $-\%$ hazardous steps - represents the percentage of total steps that were on designated hazards. The second measure $-\%$ failed hazards - represents the percentage of total hazards which a participant failed to observe (where a hazard was considered failed if a participant stepped at least once on the corresponding color during the time the hazard was displayed). We found no significant differences for either measure according to avoidance cue or gender; irrespective of cue or gender, approximately $12 \%$ of participants' steps were hazardous, and participants failed about $1 / 3$ of the hazards - demonstrating the non-trivial nature of the hazard avoidance system.

\subsection{Lap Times (Walking Speed)}

We measured participants' average lap times (which we use as a simple measure of walking speed): (a) when carrying but not using the mobile device and when walking without avoiding hazards ('normal'); (b) when carrying but not using the mobile device but when walking while avoiding hazards ('avoid'); and (c) when using the mobile device to enter text while walking and avoiding hazards ('experimental'). This range of measures allowed us to observe the impact of the experimental condition itself (normal v. avoid) as well as the impact of the task (avoid $\mathrm{v}$. experimental). We found a significant difference in lap times based on gender $\left(\mathrm{F}_{1,36}=14.45, \mathrm{p}=0.001\right)$ and on measurement scenario $\left(\mathrm{F}_{2,36}=22.58, \mathrm{p}<0.001\right)$. Avoidance cue did not appear to impact on lap times.

Women generally walked slower (avg. 12.7s) than men (avg. 11.6s): this is, perhaps, unsurprising given the physiological differences between the genders. Across all participants, an average lap time of $11.1 \mathrm{~s}$ was observed for the normal scenario, $12.0 \mathrm{~s}$ for the avoidance scenario, and $13.3 \mathrm{~s}$ for the experimental sessions (irrespective of avoidance cue used); the difference between all three of these average lap times was significant (normal v. avoid, $\mathrm{p}=0.026$; normal v. experimental, $\mathrm{p}<0.001$; and avoid $v$. experimental, $\mathrm{p}=0.001$ ). These differences highlight (a) the impact of the need to avoid hazards while mobile, and (b) the further, added, impact of completing mobile data entry tasks while mobile and avoiding hazards. In so doing, the differences stress the importance of incorporating contextually-relevant aspects such as contextual awareness into mobile evaluations of mobile technologies. 


\subsection{Task Load Ratings}

The NASA Task Load Index (TLX) assesses subjective workload according to 6 independent dimensions (see Figure 5). Each is measured on a 20 point scale; for all bar the performance rating, a lower score is better. The average ratings from our participants are shown in Figure 5.

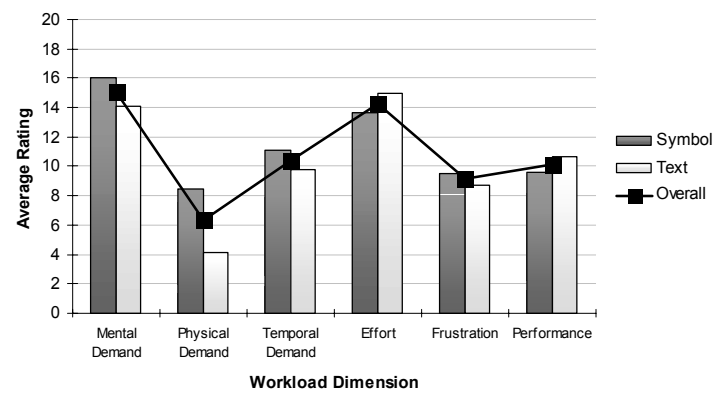

Figure 5: Workload ratings according to avoidance cue.

Neither avoidance cue nor gender had a significant impact on overall ratings. We did, however, find that avoidance cue had a significant effect on physical demand $\left(\mathrm{F}_{2,12}=5.03, \mathrm{p}=0.045\right)$, with participants exposed to the textual cue reporting a significantly lower level of physical demand. Since there was no significant difference between participants in terms of their successful hazard avoidance or lap times on the basis of avoidance cue, we find this difference in their assessment of physical demand surprising. It may be that participants translated their difficulty in achieving accurate data entry when exposed to the symbolic cue into an increased level of physical demand; further study would be required to investigate this fully. We also found that the combination of gender and avoidance cue had a significant effect on effort $\left(\mathrm{F}_{1,12}=8.54\right.$, $\mathrm{p}=0.013$ ). Specifically, females exposed to the symbolic cue rated the effort required to complete their tasks significantly lower (avg. 12.0) than females exposed to the textual cue (avg. 16.0, $\mathrm{p}=0.037$ ). This final observation accords with our original hypothesis, but seemingly contradicts all of our other observations thus far. Further investigation would be required to understand this apparent anomaly.

\section{CONCLUSIONS \& FURTHER WORK}

As we expected, we observed that avoidance cue had a significant effect on participants' ability to enter accurate text using speech; what we did not anticipate, however, was that it would be the textual avoidance cue that, on initial inspection at least, interfered less with participants' ability to complete data input tasks. The fact that the symbolic cue appeared to be harder to process (in terms of achieving accurate text entry) raises an interesting question: which cue type is most representative of real world requirements (i.e., better reflects likely real world cognitive demands)? This will undoubtedly depend on the specific context in which a user is operating in the real world, but further comparative studies with real world trials would be required to determine under which situations it may be better to use the symbolic cue rather than the textual cue. We have shown that, although they exerted a different impact on participants' ability to accurately enter text, the avoidance cues did not appear to exert differing impact in terms of successful hazard avoidance per se or task completion/lap times. This suggests that, taken in isolation, both may work equally well as facets of the hazard avoidance system; their applicability, however, will likely be determined by the task/system being evaluated.
Some of the gender-based differences are interesting; specifically, the results from male participants seemed to most consistently embody the dominance of the textual avoidance cue. Further research would be needed to investigate more fully some of the gender-based variances.

Finally, we have obviously only tested visual avoidance cues in this study; we would propose to test (1) audio cues, and (2) audio+visual cues in the future to determine their impact and usefulness when incorporated into our hazard avoidance system. At this time, we present these results as (a) further evidence of the merits of incorporating environmental awareness in mobile evaluations, and (b) an initial indication of the need to carefully consider the use of appropriate hazard cues relative to ultimate context in such studies.

\section{REFERENCES}

[1] Crease, M., Lumsden, J. and Longworth, B., 2007. A Technique for Incorporating Dynamic Paths in Lab-Based Mobile Evaluations. In Proceedings of the British HCI'2007 Conference, (Lancaster, UK, 3-7 Sept, 2007), BCS, 99-108.

[2] Hart, S.G. and Wickens, C., 1990. Workload assessment and prediction. in Booher, H.R. ed. MANPRINT: an approach to systems integration, Van Nostrand Reinhold, New York, 257 - 296.

[3] Kjeldskov, J., Skov, M.B., Als, B.S. and Høegh, R.T., 2004. Is It Worth the Hassle? Exploring the Added Value of Evaluating the Usability of Context-Aware Mobile Systems in the Field. In Proceedings of the 6th International Symposium on Mobile Human-Computer Interaction (MobileHCI'04), (Glasgow, Scotland, September 13 - 16, 2004), 61 - 73.

[4] Kjeldskov, J. and Stage, J., 2004. New Techniques for Usability Evaluation of Mobile Systems. International Journal of Human Computer Studies (IJHCS), 60(5-6). $599-620$

[5] Lumsden, J., Kondratova, I. and Durling, S., 2007. Investigating Microphone Efficacy for Facilitation of Mobile Speech-Based Data Entry. In Proceedings of the British HCI'2007 Conference, (Lancaster, UK, 3-7 Sept, 2007), BCS, 89-98.

[6] MacKenzie, I.S. and Soukoreff, R.W., 2003. Phrase Sets for Evaluating Text Entry Techniques. In Proceedings of the Human Factors in Computing Systems - CHI'2003, (Ft. Lauderdale, USA, 5 - 10 April, 2003), 754 - 755.

[7] Nielsen, C.M., Overgaard, M., Pedersen, M.B., Stage, J. and Stenild, S., 2006. It's Worth the Hassle! The Added Value of Evaluating the Usability of Mobile Systems in the Field. In Proceedings of the 4th Nordic Conference on Human-Computer Interaction: Changing Roles (NordiCHI'06), (Oslo, Norway, October 14-18, 2006), ACM Press, 272-280.

[8] Oviatt, S., 2000. Taming Recognition Errors with a Multimodal Interface. Communications of the ACM, 43(9). $45-51$.

[9] Pirhonen, P., Brewster, S.A. and Holguin, C., 2002. Gestural and Audio Metaphors as a Means of Control in Mobile Devices. In Proceedings of the ACM-CHI'2002, (Minneapolis, MN, April 20 - 25, 2002), ACM Press Addison-Wesley, 291 - 298. 